\title{
SCL-90 R: Distrés psicológico, género y conductas de riesgo*
}

SCL-90 R: Psychological Distress, Gender and Risky Behaviors

Recibido: octubre 11 de 2010 | Revisado: noviembre 24 de 201 | Aceptado: junio 20 de 2012

\author{
ARMANDO RIVERA-LEDESMA ** \\ Nancy Patricia Caballero Suárez \\ Ivonne Nalliely Pérez SÁNCHez \\ Universidad de Londres, Ciudad de México, México \\ María Montero-LÓPez LeNA *** \\ Universidad Nacional Autónoma de México, México
}

\section{RESUMEN}

Con base en una muestra probabilística de 466 estudiantes universitarios mexicanos, se explora la relación entre distrés psicológico y conductas de riesgo (consumo de sustancias, conductas sexuales, y ambas), en hombres y mujeres. Los resultados indican que el distrés psicológico presenta diferencias con mayor frecuencia en el caso de las mujeres que presentan consumo de drogas, tabaco y alcohol, o conductas que mezclan sexo y alcohol, y sexo y consumo de drogas, que en el caso de los hombres. Los hombres con actividad sexual ocasional presentan mayor distrés que aquellos que no presentan esta conducta. Como punto de partida, se explora la estructura factorial del SCL-90-R, coincidiendo en los resultados con los obtenidos por investigadores de otras latitudes; al parecer, el análisis factorial no soporta la estructura propuesta por los autores del instrumento.

Palabras clave autores

SCL-90-R, distrés, género, consumo de sustancias, conductas sexuales de riesgo.

Palabras clave descriptores

Psicología de la Salud, investigación cuantitativa, Psicometría, México 105-118.

* Artículo de investigación. Agradecimientos: Agradecemos el apoyo otorgado por la Universidad de Londres, Campus Ciudad de México, al proyecto "Conductas sexuales de riesgo y consumo de sustancias en estudiantes universitarios" realizado entre los meses de junio y septiembre del 2010.

** Coordinación de Investigación y Producción Científica. Dirección para correspondencia: Plaza de Luis Cabrera No. 9 Col. Roma, Delegación Cuauhtémoc. México, D.F. 06700, Teléfono: (55) 52077405. E-mails: armandoriveral@hotmail.com,nancygansy@hotmail.com, ivnnaey@hotmail.com

**** Facultad de Psicología, División de Estudios de Posgrado, Cubículo 7, Av. Universidad 3004, Copilco Universidad, Ciudad Universitaria, México, D.F. 04510, Teléfono: (55) 5622-2241. E-mail: monterol@unam.mx

\begin{abstract}
A B S T R A C T of the instrument.

Key words authors

SCL-90-R, distress, gender, substance abuse, sexual risk behaviors.

Key words plus

Health Psychology, Quantitative Research, Psychometry, Mexico.
\end{abstract}

Based on a probability sample of 466 Mexican college students, we want to find out if there is a relationship between psychological distress and risky behavior (drug-consuming, sexual behavior or both) between men and women. Results show that psychological distress presents more differences with women who consume drugs, tobacco and alcohol and who present behaviors which combine sex and alcohol more than men. Men with occasional sexual activity preset more distress than those who don't show this behavior. To begin, we explore the factorial structure of SCL-90-R observing the same results of those obtained by other researchers. It seems that the factor analysis doesn't support the structure proposed by the authors 


\section{Antecedentes}

Uno de los sectores más vulnerables de la población es el de la gente joven, comprendida entre los 18 y los 30 años de edad, en relación con hábitos y conductas que suelen poner en peligro la vida. Parece ser que el distrés psicológico juega un importante papel en la generación de conductas de riesgo para la salud; se ha destacado que los jóvenes universitarios están expuestos al desarrollo de nuevas relaciones de intimidad y al despliegue de una sexualidad emergente que puede generar tensiones importantes (Schulenberg, Bryant \& O’Malley, 2004), capaces de potenciar el riesgo de contagio de enfermedades de transmisión sexual (Antón \& Espada, 2009) o el consumo de sustancias adictivas.

Se ha informado, por ejemplo, que los trastornos por consumo de sustancias (CS) se concentran en buena medida entre los 15 y los 30 años de edad, y que representan un problema social de amplias dimensiones en México (Medina-Mora et al., 2003), que cada vez con mayor claridad va incorporando a hombres y mujeres prácticamente en la misma medida, aunque con diferencias en los patrones de consumo (Ortíz, Soriano, Meza, Martínez \& Galván, 2006; Villatoro et al., 2006). Otro grave problema altamente prevalente en este sector etario es la infección por VIH; se ha estimado que el $45 \%$ de todas las infecciones por VIH en el mundo han ocurrido en sujetos entre los 15 y los 24 años de edad (Antón \& Espada, 2009). Por otro lado, se ha destacado la importancia de ciertos estados comórbidos caracterizados por la asociación entre trastornos mentales y CS, señalándose el significativo incremento en el riesgo de suicidio evidenciado por esta relación (Caraveo-Anduaga \& Colmenares, 2002). Otra comorbilidad significativa es la existente entre conductas sexuales de riesgo (CSR) y trastornos mentales. Recientemente, se ha señalado, por ejemplo, el trastorno por hipersexualidad (hypersexual disorder, término sugerido por el subgrupo de trabajo para parafilias del DSM-V; Kaplan \& Krueger, 2010), como una CSR asociada a depresión mayor, distimia, abuso de sustancias y ansiedad fóbica, con capacidad para incrementar la posibilidad de contagio de enfermedades de transmisión sexual, entre ellas el VIH.

Semple, Strathdee, Zians y Patterson (2010), investigando la relación entre anfetaminas, eventos de la vida y el riesgo sexual en sujetos heterosexuales, propusieron un modelo de escape cognitivo mediante el cual los usuarios de metanfetaminas pueden entablar CSR para escapar de los afectos negativos asociados a los estresores negativos de la vida, lo cual ha sido evidenciado por contribuciones de otros investigadores (Halkitis, Mukherjee \& Palamar, 2007; Semple, Patterson \& Grant, 2002 citados por Semple et al., 2010). Así, se ha sugerido que el distrés psicológico es una variable que puede minar la intención de protección contra el VIH o que puede estar asociada a CSR (Brodbeck, Vilén, Bachmann, Znoj \& Alsaker, 2010), aunque al parecer esta asociación ha sido documentada en mujeres; Brodbeck et al. (2010) aportaron evidencia con respecto al significativo papel predictor del distrés psicológico con respecto a la generación de CSR en mujeres entre los 16 y los 24 años de edad.

Ahora bien, una de las medidas más exploradas en distintas latitudes para la medición del distrés psicológico es el Derogatis Sympton ChecklistRevised ([SCL-90-R], Inventario de Síntomas de Derogatis-Revisado). El SCL-90-R fue desarrollado por el propio Derogatis y sus colaboradores (Derogatis, 1994; Derogatis, Lipman \& Covi, 1973) como una medida de distrés psicológico (malestar psicológico actual). Actualmente está disponible en más de 26 lenguas, habiéndose adaptado al español para distintos contextos iberoamericanos (v. gr., Caparrós, Villar, Ferrer \& Viñas, 2007; Cruz, López, Blas, González \& Chávez, 2005; Gempp \& Avendaño, 2008), y las características específicas del instrumento son ampliamente conocidas; en particular, Gempp y Avendaño (2008) han desarrollado una excelente descripción.

La consistencia interna del SCL-90-R, tanto para el total de la escala como para cada una de sus nueve dimensiones, ha generado valores entre 0.7 y 0.9 en distintas muestras (v. gr., estudiantes universitarios y pacientes con diagnostico psiquiátrico, con enfermedades médicas como cáncer, dolor crónico, desorden temporo-mandibular, etc.; 
Carrasco, Sánchez, Ciccotelli \& Del Barrio, 2003; Lara, Espinosa, Cárdenas, Fócil \& Cavazos, 2005), mostrando una adecuada validez concurrente y predicativa al emplear como criterios otros instrumentos de evaluación clínica (v. gr., MMPI, escalas clínicas de depresión y ansiedad), escalas de tamizaje y diagnósticos psiquiátricos, entre otros. Sin embargo, no parece existir un acuerdo entre los resultados de diferentes investigadores y en distintas latitudes con respecto a su estructura factorial; esta no ha podido ser adecuadamente documentada, habiéndose obtenido estructuras discordantes con la propuesta originalmente por Derogatis y sus colaboradores (cf., Bonynge, 1993; Caparrós et al., 2007; Schwarzwald, Weisenberg \& Solomon, 1991; Steer, Clark \& Ranieri, 1994). Se ha sugerido (Gempp \& Avendaño, 2008) que al parecer esta técnica estadística puede ser poco apropiada para un instrumento clínico cuyo comportamiento factorial puede variar según la prevalencia de síntomas de la población en que es aplicado, argumentándose que quizá lo mejor es obviar esta cuestión apelando a su alta consistencia interna y su alta validez convergente, concurrente y predictiva.

El presente trabajo tuvo como objetivo general describir la relación entre distrés psicológico y conductas sexuales de riesgo y consumo de sustancias, en hombres y mujeres mexicanos. Como paso previo a este abordaje, se explora la estructura factorial del SCL-90-R en una muestra probabilística de estudiantes universitarios mexicanos con miras a evaluar si los resultados coinciden con la estructura propuesta por Derogatis y sus colaboradores, y si, en todo caso, los resultados son semejantes a los obtenidos en otras latitudes de Latinoamérica y España. Finalmente, se explora la relación entre distrés psicológico y CS, CSR y CS-CSR sobre la hipótesis de que entre individuos con alto distrés la proporción de sujetos que recurren a CS, CSR y CS-CSR es mayor que la de aquellos que no lo hacen. Con este esfuerzo, la presente investigación se suma a la necesidad de contribuir a la realización de estudios transculturales en localidades de habla hispana, que permitan conocer mejor tanto al SCL-90-R como la expresión sintomatológica de las distintas poblaciones.

\section{Método}

\section{Muestra}

Mediante muestreo aleatorio estratificado con asignación proporcional, se seleccionaron 466 estudiantes universitarios de un total de 3.249 alumnos registrados en las carreras promovidas por una universidad privada de Ciudad de México. Para la selección se partió por niveles en el siguiente orden: campus, turnos, carreras o disciplinas y salones. Las características sociodemográficas de la muestra se describen en la Tabla 1.

\section{Procedimiento}

A los estudiantes seleccionados se les invitó a colaborar informándoles que su participación sería voluntaria y que los datos obtenidos serían tratados en forma confidencial. La aplicación se realizó de forma colectiva durante el tiempo de clase y directamente en las aulas dispuestas por la universidad. A fin de resolver dudas y vigilar el proceso de campo, un investigador permaneció en el aula durante la aplicación del cuestionario. Los datos resultantes fueron capturados y analizados en el sistema PASW Statistics 18.

\section{Instrumentos}

\section{SCL-90-R}

Para esta investigación se consideró la versión de la escala traducida y validada por Cruz et al. (2005). Esta versión es muy similar a la empleada por Casullo (2004a, 2004b) con sujetos argentinos, diferenciándose por la adaptación de términos de uso cotidiano (de términos como tren, ómnibus, subterráneos a términos como tren, microbús, pesero o metro) en el reactivo 47. La escala tuvo una consistencia interna total y por dimensiones entre 0.7 y 0.85 . Está compuesta de 90 reactivos agrupados en nueve dimensiones clínicas primarias: Somatización, Obsesiones/ Compulsiones, Sensibilidad Interpersonal, Depresión, Ansiedad, Hostilidad, Ansiedad Fóbica, 


\section{TABLA 1}

Características sociodemográficas de la muestra

\begin{tabular}{|c|c|c|c|}
\hline $\begin{array}{c}\text { Sexo: } \\
\text { Hombres: } 184(39.5 \%) \\
\text { Mujeres: } 282(60.5 \%) \\
\text { Total: } 466(100 \%)\end{array}$ & & $\begin{array}{c}\text { Edad: } \\
22.3 \text { años }(D E=3.7) \\
21.8 \text { años }(D E=3.8) \\
22 \text { años }(D E=3.8)\end{array}$ & \\
\hline Distribución por Carrera & $f(\%)$ & Distribución por ciclo escolar & $f(\%)$ \\
\hline Psicología & $111(23.8)$ & $1^{\mathrm{o}}$ & $39(8.4)$ \\
\hline Derecho & $75(16.1)$ & $2^{\mathrm{O}}$ & $152(32.6)$ \\
\hline Gastronomía & $50(10.7)$ & $3^{\mathrm{o}}$ & $23(4.9)$ \\
\hline Administración & $39(8.4)$ & $4^{\mathrm{O}}$ & $75(16.1)$ \\
\hline Informática & $32(6.9)$ & 50 & $54(11.6)$ \\
\hline Diseño gráfico & $30(6.4)$ & $6^{\mathrm{e}}$ & $47(10.1)$ \\
\hline Administración turística & $27(5.8)$ & $7^{\circ}$ & $18(3.9)$ \\
\hline Contaduría & $25(5.4)$ & $8^{\circ}$ & $48(10.3)$ \\
\hline Mercadotecnia & $25(5.4)$ & 90 & $10(2.1)$ \\
\hline Diseño de modas & $23(4.9)$ & & \\
\hline Com. Multimedia & $21(4.5)$ & & \\
\hline Actuación & $5(1.1)$ & & \\
\hline Finanzas & $3(0.6)$ & & \\
\hline
\end{tabular}

Fuente: elaboración propia.

TABLA 2

Análisis de diferencias significativas por género en la muestra de México 2010

\begin{tabular}{|c|c|c|c|c|c|c|c|}
\hline & \multicolumn{2}{|c|}{ Hombres } & \multicolumn{2}{|c|}{ Mujeres } & \multirow{2}{*}{$t$} & \multirow{2}{*}{$g l$} & \multirow{2}{*}{$p$} \\
\hline & $M$ & $D E$ & $M$ & $D E$ & & & \\
\hline Somatización & 0.54 & 0.58 & 0.75 & 0.62 & 3.723 & 455 & 0.000 \\
\hline Obs. / Comp. & 0.70 & 0.59 & 0.89 & 0.74 & 3.002 & 433.2 & 0.003 \\
\hline Depresión & 0.48 & 0.56 & 0.67 & 0.64 & 3.387 & 413.9 & 0.001 \\
\hline IGS & 0.51 & 0.49 & 0.61 & 0.49 & 2.275 & 455 & 0.023 \\
\hline IMP & 1.49 & 0.49 & 1.62 & 0.46 & 2.352 & 452 & 0.019 \\
\hline $\mathrm{TP}$ & 27.07 & 19.52 & 30.95 & 19.15 & 2.101 & 455 & 0.036 \\
\hline
\end{tabular}

Nota. Solo se incluyen diferencias significativas.

Fuente: elaboración propia.

Ideación Paranoide, Psicoticismo, y tres índices globales: Índice de Severidad o Gravedad Global (ISG), Total de Síntomas Positivos (TSP) e Índice de Malestar Positivo (IMP).

\section{Conductas sexuales de riesgo}

Se estructuró un cuestionario compuesto de reactivos previamente validados sobre conductas sexuales de riesgo con base en los trabajos de Uribe (2007) y Meave (2008); en ambos instrumentos la consis- tencia interna medida con el método de Cronbach fue de 0.8 .

\section{Consumo de sustancias}

Se utilizó el instrumento diseñado por Rodríguez y Hernández (2005), validado en estudiantes universitarios para la detección del consumo de drogas lícitas (alcohol y tabaco) e ilícitas (cocaína, éxtasis, pastas, y otras) así como delitos y creencias. Su consistencia interna osciló entre índices alpha de 0.66 y 0.77 . 


\section{Resultados}

\section{Estructura factorial}

Se estimaron los índices de adecuación muestral mediante la prueba de esfericidad de Bartlett y la medida de Kaiser-Meyer-Olkin con resultados que justificaron lo procedente del análisis factorial (0.940, $p<0.001$, respectivamente). Los resultados obtenidos mediante el método de componentes principales con rotación VARIMAX y valores Eigen mayores a 1 no permitieron replicar la estructura propuesta por Derogatis. La estructura resultante arrojó 18 factores que explicaron el $63.1 \%$ de la varianza, lo cual fue ampliamente discordante con lo esperado. Los cinco primeros factores hallados fueron los más robustos con 13, 9, 9, 8 y 4 reactivos; el resto de los factores incluyeron 3 o menos; los cinco primeros factores sugerían estar midiendo Depresión como suele ser encontrada en adolescentes (reactivos, 30, 29, 34, 11, 3, 36, 31, 20, 33, 76, 54, 18 y 77), Síntomas físicos (reactivos 42, 58, 52, $39,17,53,45,57$ y 49), Culpa con ideas paranoides (reactivos 85, 43, 82, 80, 35, 73, 83, 37 y 62), Pérdida de energía física y mental (reactivos 9, 10, 55, 56, 28, 14, 46, 32 y 38) y Fobias específicas (47, 50, 25 y 13). Dada la falta de acuerdo entre los diferentes investigadores con respecto a la estructura factorial del SCL-90-R, se decidió continuar el análisis considerando la estructura originalmente propuesta por Derogatis y adaptada en sujetos mexicanos por
Cruz et al. (2005). Así, se compararon las medias obtenidas por hombres y mujeres en cada una de las dimensiones primarias y los índices globales; solo tres dimensiones y los tres índices reportaron diferencias significativas. En general, los hombres calificaron más bajo que las mujeres en todas las dimensiones e índices, aunque no siempre de manera significativa. La Tabla 2 muestra estos resultados.

En virtud de que las coincidencias entre medias por sexo eran parciales, se decidió continuar los análisis considerando a cada sexo en forma independiente. Así, se exploró la correlación entre las distintas dimensiones con datos separados por sexo con los resultados descritos en la Tabla 3. Como es dado observar, las correlaciones resultaron en su gran mayoría superiores a 0.5 en ambos sexos, lo cual aporta evidencia acerca de la unidad conceptual (distrés) entre ellas. Un análisis detenido entre las correlaciones de ambos grupos parece mostrar que la escala se comporta en términos muy similares en ambas submuestras.

\section{Confiabilidad}

La consistencia interna de la escala calculada por el método de Cronbach en su versión original fue adecuada para todas las subescalas: Somatización: 0.85; Obsesiones/ Compulsiones: 0.85; Sensibilidad interpersonal: 0.81; Depresión: 0.88; Ansiedad: 0.77; Hostilidad: 0.74; Ansiedad fóbica: 0.73; Ideación paranoide: 0.74 y Psicoticismo: 0.83. La consistencia interna general fue de 0.96 .

TABLA 3

Correlaciones bivariadas entre dimensiones primarias del SCL-90-R

\begin{tabular}{cccccccccc}
\hline & So & O-C & Se & D & A & H & AF & IP & Ps \\
\hline So & & 0.65 & 0.62 & 0.72 & 0.81 & 0.55 & 0.7 & 0.57 & 0.72 \\
O-C & 0.65 & & 0.71 & 0.76 & 0.76 & 0.6 & 0.6 & 0.65 & 0.72 \\
Se & 0.57 & 0.65 & & 0.78 & 0.78 & 0.66 & 0.69 & 0.77 & 0.8 \\
D & 0.66 & 0.74 & 0.76 & & 0.83 & 0.69 & 0.69 & 0.73 & 0.8 \\
A & 0.74 & 0.73 & 0.72 & 0.69 & & 0.66 & 0.74 & 0.65 & 0.81 \\
H & 0.57 & 0.57 & 0.58 & 0.62 & 0.58 & & 0.59 & 0.58 & 0.66 \\
AF & 0.5 & 0.52 & 0.57 & 0.5 & 0.59 & 0.42 & & 0.62 & 0.77 \\
IP & 0.55 & 0.67 & 0.77 & 0.7 & 0.65 & 0.61 & 0.5 & & 0.74 \\
Ps & 0.7 & 0.68 & 0.74 & 0.75 & 0.73 & 0.55 & 0.58 & 0.77 & \\
\hline
\end{tabular}

Nota. Hombres: Triángulo superior. Mujeres: Triángulo inferior. Fuente: elaboración propia. 


\section{Hombres}

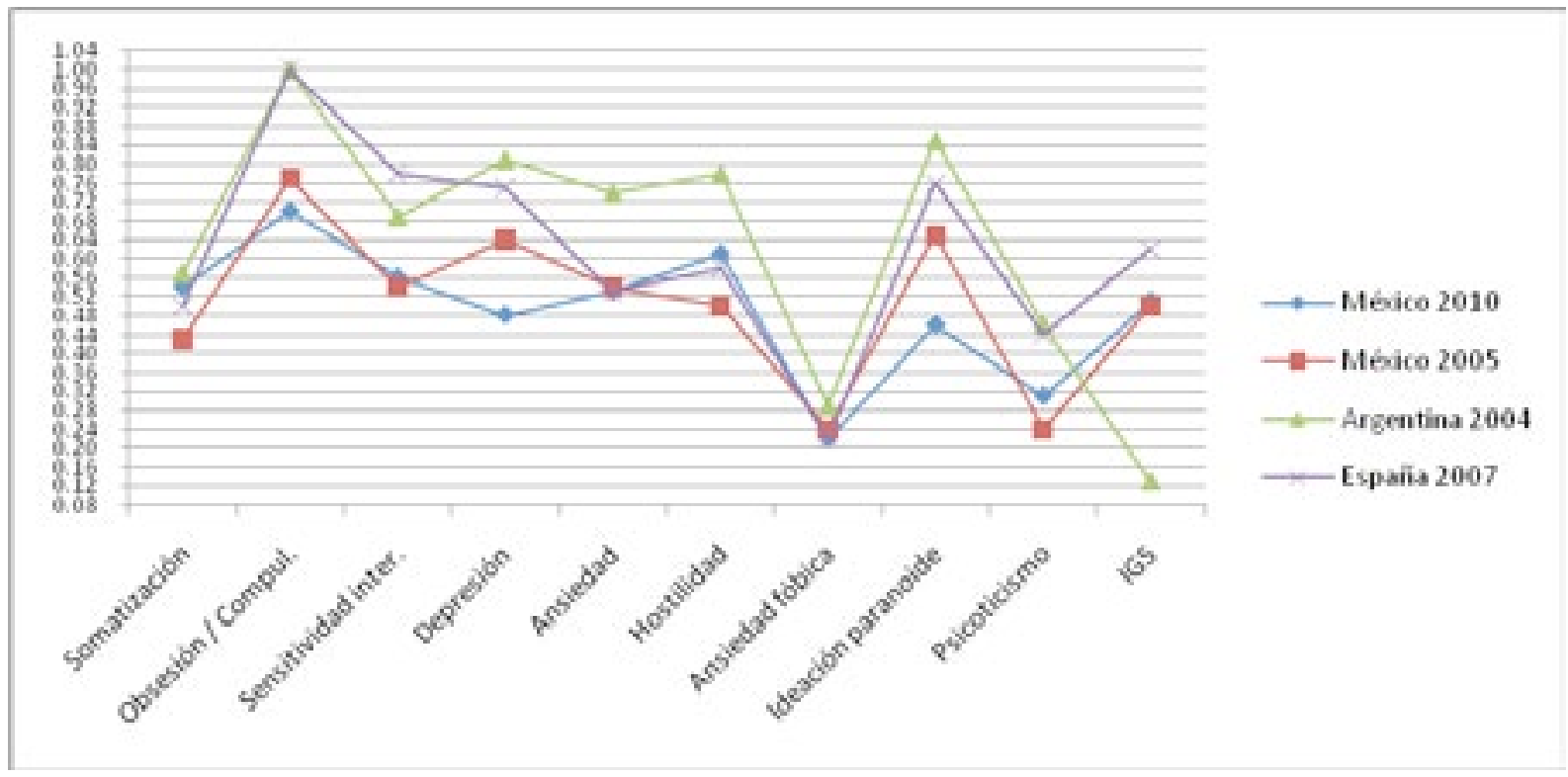

Figura 1. Medias para dimensiones primarias e Índice de Severidad Global SCL-90-R.

Fuente: Caparrós et al. (2007); Casullo (2004a); Cruz et al. (2005).

\section{Mujeres}

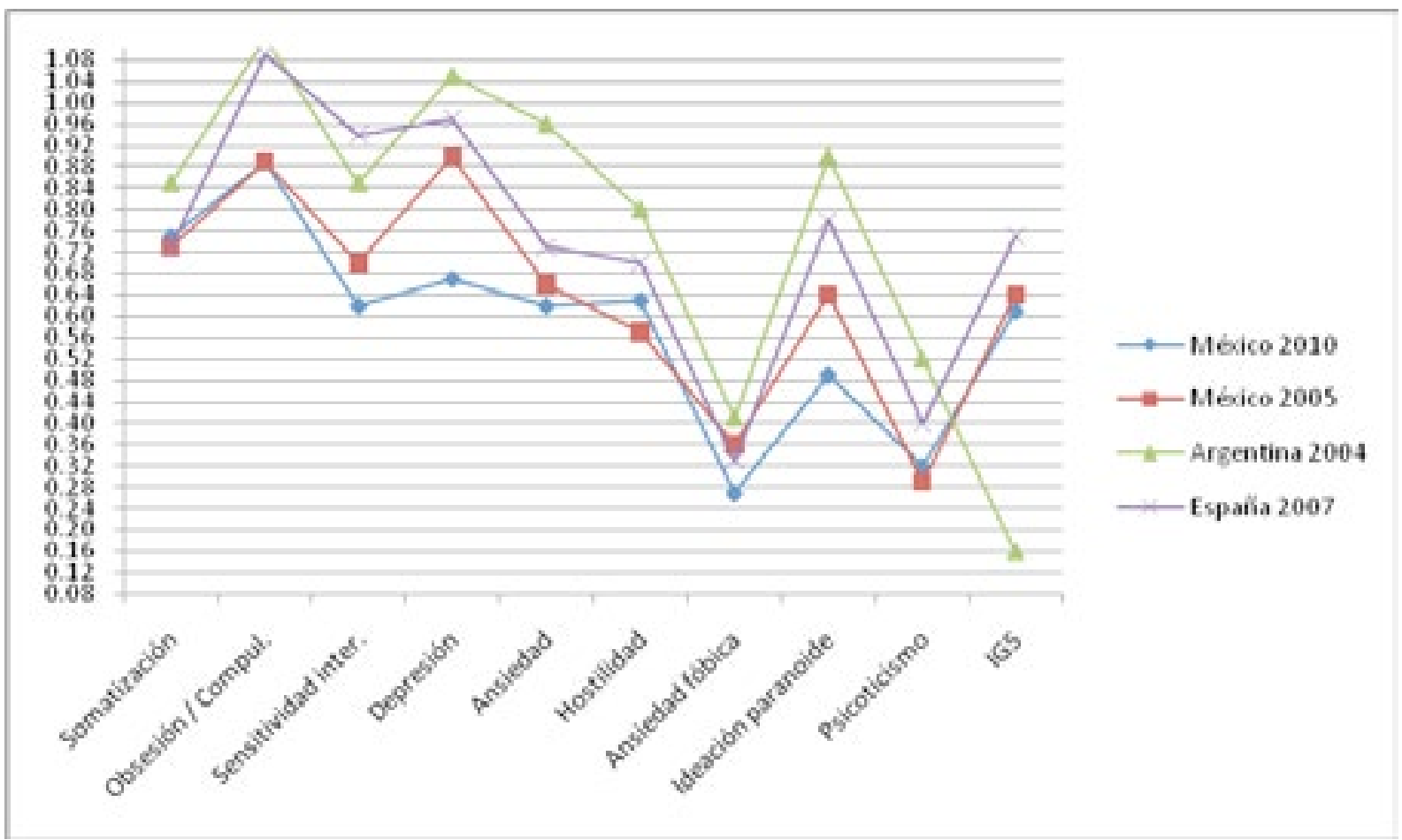

Figura 2. Medias para Dimensiones primarias e Índice de Severidad Global SCL-90-R.

Fuente: Caparrós et al. (2007); Casullo (2004a); Cruz et al. (2005). 
TABLA 4

Diferencias significativas entre la muestra México 2010 y las muestras

\begin{tabular}{|c|c|c|c|c|c|c|}
\hline & \multicolumn{3}{|c|}{ Hombres } & \multicolumn{3}{|c|}{ Mujeres } \\
\hline & $\begin{array}{c}\text { México } \\
2005\end{array}$ & $\begin{array}{c}\text { Argentina } \\
2004\end{array}$ & $\begin{array}{c}\text { España } \\
2007\end{array}$ & $\begin{array}{c}\text { México } \\
2005\end{array}$ & $\begin{array}{c}\text { Argentina } \\
2004 \\
\end{array}$ & $\begin{array}{c}\text { España } \\
2007\end{array}$ \\
\hline Somatización & $* *$ & $=$ & $=$ & $=$ & $* *$ & $=$ \\
\hline Obs. / Comp. & $=$ & $* *$ & $* *$ & $=$ & $* *$ & $* *$ \\
\hline Sensibilidad & $=$ & $* *$ & $* *$ & $*$ & $* *$ & $* *$ \\
\hline Depresión & $* *$ & $* *$ & $* *$ & $* *$ & $* *$ & $* *$ \\
\hline Ansiedad & $=$ & $* *$ & $=$ & $=$ & $* *$ & $* *$ \\
\hline Hostilidad & $*$ & $* *$ & $=$ & $=$ & $* *$ & $=$ \\
\hline Ans.fóbica & $=$ & $* *$ & $=$ & $* *$ & $* *$ & $=$ \\
\hline Ideación para. & $* *$ & $* *$ & $* *$ & $* *$ & $* *$ & $* *$ \\
\hline Psicoticismo & $=$ & $* *$ & $* *$ & $=$ & $* *$ & $* *$ \\
\hline IGS & $=$ & $* *$ & $* *$ & $=$ & $* *$ & $* *$ \\
\hline
\end{tabular}

Nota. $=$ Sin diferencias significativas prueba $t$ para una sola muestra. $* p \leq 0.05 . * * \leq \leq 0.01$.

Fuente: Caparrós et al. (2007); Casullo (2004a); Cruz et al. (2005).

\section{Análisis del perfil dimensional contra otras investigaciones}

Por otro lado, se exploró la posible coincidencia de los resultados obtenidos con respecto a otras investigaciones realizadas tanto en México como en otras latitudes iberoamericanas. Al efecto, se compararon las medias de hombres y mujeres de la muestras de Argentina (Casullo, 2004a); España (Caparrós et al., 2007) y México (Cruz et al., 2005), con respecto a la obtenida en el presente trabajo; no se incluyó la muestra chilena porque no incluía información por sexos (Foguras 1 y 2 ).

Los hombres parecen puntuar más bajo que las mujeres en las cuatro muestras y en todas las dimensiones, lo cual es consistente con los resultados reportados anteriormente en esta investigación. Por otro lado, las gráficas sugieren que las dimensiones asumen un comportamiento similar en las cuatro muestras; en general parecen crecer y decrecer con cierta uniformidad, sin bien las muestras de España y Argentina podrían distanciarse de las dos muestras mexicanas en cuanto a la "intensidad o fuerza" (es decir, un mayor puntaje que sin embargo conserva la misma tendencia), de los síntomas.

Por ejemplo, las dos muestras mexicanas no presentan diferencias significativas en el $40 \%$ de sus dimensiones; sin embargo, la investigada en
2010 muestra diferencias significativas con prácticamente todas las dimensiones de la explorada en Argentina por Casullo (2004a) y con aproximadamente el $70 \%$ de la española (Caparrós et al., 2007) (Tabla 4). Aunque se conserva la tendencia general de cada dimensión, la fuerza con que se expresa en cada cultura parece distinta. Estas diferencias podrían deberse a discrepancias culturales centradas en distintos modos de expresividad de los síntomas o divergencias cualitativas en la intensidad con la cual se afrontan o experimentan situaciones estresantes.

\section{Valores percentiles}

Se construyeron valores percentiles para facilitar la comparación de resultados, tras la aplicación del SCL-90-R. Se decidió calcular percentiles para hombres y mujeres en acuerdo a lo reportado más arriba con los resultados descritos en las Tablas 5a y 5 b.

El Grafico 3 muestra la comparación entre los percentiles obtenidos en los estudiantes mexicanos de la presente investigación y los obtenidos por Caparrós et al. (2007), en España en una muestra similar de alumnos. Como puede observarse ambas muestras son similares, aunque los mexicanos tienden a puntuar ligeramente más bajo. 
TABLA 5A

Percentiles para las dimensiones primarias e índices globales

\begin{tabular}{ccccccccccccc}
\hline & & & \multicolumn{1}{c}{ Hombres } & & & & \\
\hline Centiles & So & O-C & Se & D & A & H & AF & IP & Ps & IGG & TSP & IMP \\
\hline 5 & 0.00 & 0.00 &. & & & & & & & 0.05 & 5 & 0.90 \\
10 & 0.08 & 0.10 & 0.00 & & 0.00 & 0.00 & & & & 0.08 & 7 & 1.00 \\
15 & 0.08 & 0.10 & 0.11 & 0.00 & 0.10 & 0.17 & & & & 0.12 & 8 & 1.03 \\
20 & 0.17 & 0.20 & 0.11 & 0.08 & 0.10 & 0.17 & & & & 0.15 & 11 & 1.08 \\
25 & 0.17 & 0.30 & 0.11 & 0.08 & 0.20 & 0.17 & & & & 0.20 & 13 & 1.13 \\
\hline 30 & 0.17 & 0.40 & 0.22 & 0.15 & 0.20 & 0.17 & & 0.00 & & 0.22 & 15 & 1.19 \\
35 & 0.25 & 0.40 & 0.22 & 0.15 & 0.30 & 0.33 & & 0.17 & & 0.25 & 16 & 1.25 \\
40 & 0.25 & 0.50 & 0.33 & 0.15 & 0.30 & 0.33 & & 0.17 & & 0.28 & 18 & 1.29 \\
45 & 0.33 & 0.50 & 0.33 & 0.23 & 0.30 & 0.33 & & 0.17 & 0.00 & 0.30 & 21 & 1.35 \\
50 & 0.33 & 0.60 & 0.44 & 0.31 & 0.40 & 0.33 & & 0.33 & 0.10 & 0.34 & 23 & 1.40 \\
\hline 55 & 0.42 & 0.60 & 0.44 & 0.31 & 0.50 & 0.50 & & 0.33 & 0.20 & 0.39 & 25 & 1.44 \\
60 & 0.42 & 0.70 & 0.56 & 0.46 & 0.50 & 0.50 & 0.00 & 0.33 & 0.20 & 0.42 & 27 & 1.50 \\
65 & 0.50 & 0.80 & 0.56 & 0.46 & 0.60 & 0.50 & 0.17 & 0.50 & 0.20 & 0.50 & 29 & 1.58 \\
70 & 0.58 & 0.90 & 0.67 & 0.54 & 0.60 & 0.67 & 0.17 & 0.67 & 0.30 & 0.58 & 32 & 1.69 \\
75 & 0.67 & 1.10 & 0.78 & 0.62 & 0.70 & 0.83 & 0.33 & 0.67 & 0.40 & 0.68 & 35 & 1.82 \\
\hline 80 & 0.92 & 1.20 & 0.89 & 0.77 & 0.80 & 1.00 & 0.33 & 0.83 & 0.50 & 0.76 & 40 & 1.92 \\
85 & 1.25 & 1.40 & 1.00 & 1.00 & 1.00 & 1.17 & 0.50 & 1.17 & 0.60 & 0.89 & 45 & 2.07 \\
90 & 1.42 & 1.50 & 1.22 & 1.38 & 1.20 & 1.50 & 0.83 & 1.33 & 1.00 & 1.16 & 58 & 2.23 \\
95 & 1.75 & 2.00 & 2.00 & 1.69 & 1.60 & 2.00 & 1.17 & 1.67 & 1.80 & 1.71 & 74 & 2.36 \\
100 & 3.42 & 3.40 & 3.22 & 3.23 & 3.10 & 3.17 & 3.50 & 3.33 & 3.20 & 3.24 & 90 & 3.18 \\
\hline
\end{tabular}

Fuente: elaboración propia.

TABLA 5B

Percentiles para las dimensiones primarias e índices globales

\begin{tabular}{ccccccccccccc}
\hline & & & \multicolumn{1}{c}{ Mujeres } & & & & & \\
\hline Centiles & So & O-C & Se & D & A & H & AF & IP & Ps & IGG & TSP & IMP \\
\hline 5 & 0.00 & 0.00 & & 0.00 & 0.00 & & & & & 0.09 & 7 & 1.00 \\
10 & 0.16 & 0.10 & 0.00 & 0.08 & 0.10 & & & & & 0.13 & 9 & 1.05 \\
15 & 0.17 & 0.20 & 0.11 & 0.15 & 0.19 & 0.00 & & & & 0.17 & 12 & 1.12 \\
20 & 0.25 & 0.28 & 0.11 & 0.15 & 0.20 & 0.17 & & & & 0.21 & 14 & 1.21 \\
25 & 0.33 & 0.30 & 0.19 & 0.23 & 0.30 & 0.17 & & & & 0.24 & 16 & 1.29 \\
\hline 30 & 0.42 & 0.40 & 0.22 & 0.28 & 0.30 & 0.33 & & & & 0.28 & 18 & 1.34 \\
35 & 0.42 & 0.40 & 0.33 & 0.31 & 0.30 & 0.33 & & 0.00 & 0.00 & 0.32 & 19 & 1.39 \\
40 & 0.50 & 0.50 & 0.33 & 0.38 & 0.40 & 0.33 & & 0.17 & 0.06 & 0.35 & 21.60 & 1.46 \\
45 & 0.58 & 0.60 & 0.44 & 0.38 & 0.40 & 0.50 & & 0.17 & 0.10 & 0.43 & 24.55 & 1.51 \\
50 & 0.58 & 0.70 & 0.44 & 0.46 & 0.50 & 0.50 & 0.00 & 0.33 & 0.10 & 0.49 & 27 & 1.56 \\
\hline 55 & 0.67 & 0.80 & 0.56 & 0.54 & 0.60 & 0.50 & 0.17 & 0.33 & 0.20 & 0.56 & 30 & 1.63 \\
60 & 0.75 & 0.90 & 0.56 & 0.65 & 0.60 & 0.67 & 0.17 & 0.33 & 0.20 & 0.59 & 32 & 1.68 \\
65 & 0.83 & 1.00 & 0.67 & 0.77 & 0.70 & 0.67 & 0.33 & 0.50 & 0.30 & 0.65 & 35 & 1.77 \\
70 & 0.92 & 1.13 & 0.78 & 0.85 & 0.80 & 0.83 & 0.33 & 0.67 & 0.40 & 0.74 & 38 & 1.82 \\
75 & 1.00 & 1.40 & 1.00 & 0.92 & 0.90 & 1.00 & 0.33 & 0.83 & 0.50 & 0.85 & 42 & 1.93 \\
\hline 80 & 1.17 & 1.60 & 1.00 & 1.08 & 1.00 & 1.00 & 0.50 & 1.00 & 0.60 & 0.97 & 47 & 2.03 \\
85 & 1.33 & 1.70 & 1.11 & 1.31 & 1.10 & 1.17 & 0.67 & 1.17 & 0.70 & 1.07 & 53.15 & 2.14 \\
90 & 1.67 & 1.90 & 1.44 & 1.62 & 1.30 & 1.33 & 0.83 & 1.35 & 1.01 & 1.26 & 60.10 & 2.22 \\
95 & 2.09 & 2.30 & 2.00 & 2.16 & 1.70 & 2.00 & 1.17 & 1.83 & 1.40 & 1.72 & 71.05 & 2.45 \\
100 & 3.17 & 3.60 & 3.44 & 3.23 & 3.00 & 3.00 & 2.67 & 3.00 & 2.60 & 2.59 & 89 & 3.08 \\
\hline
\end{tabular}

Fuente: elaboración propia. 


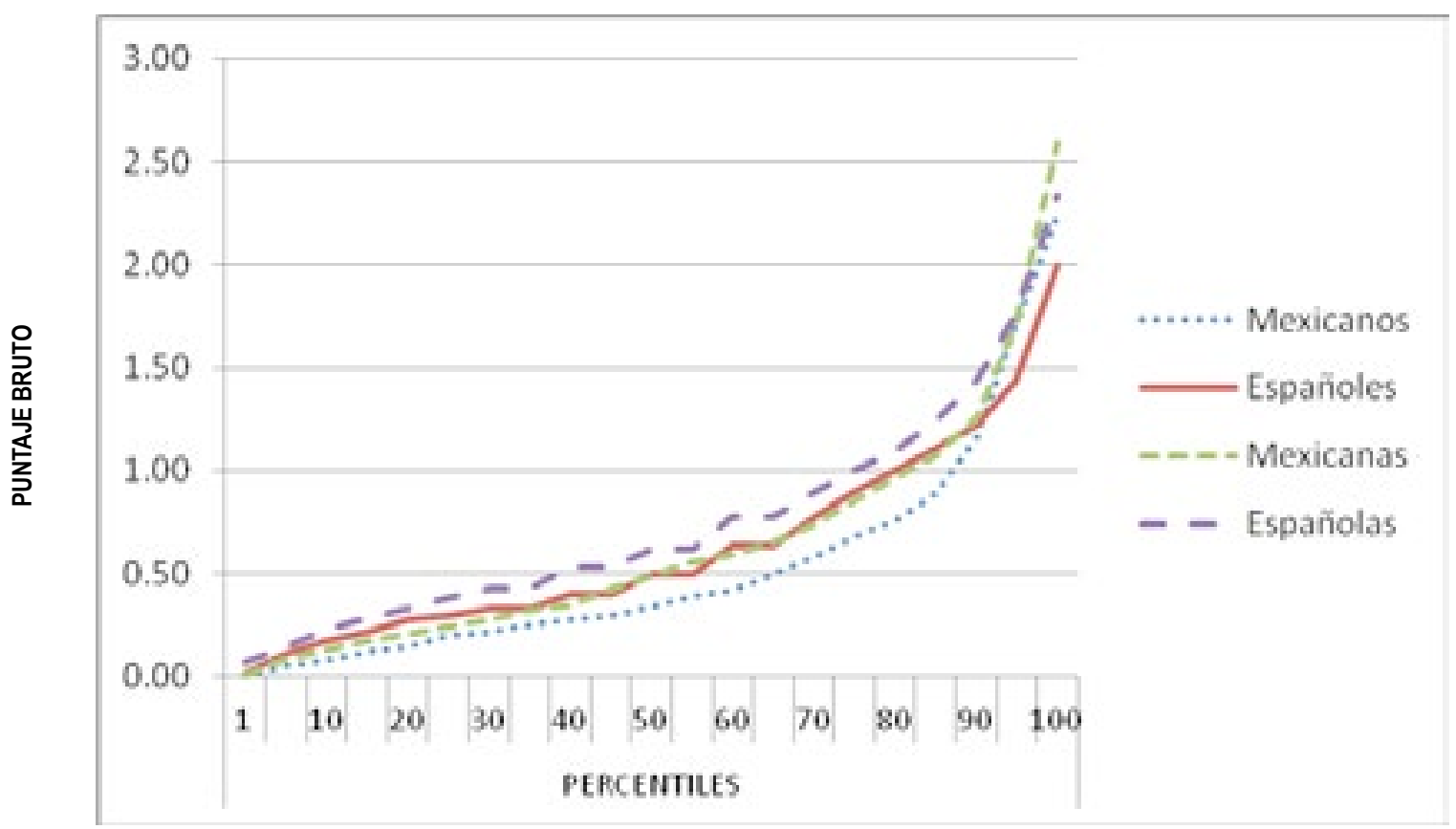

Figura 3. Comparativo de percentiles del Índice de Severidad Global SCL-90-R.

Nota. Muestras de España 2007 y México 2010.

Fuente: Caparrós et al. (2007).

Distrés psicológico, consumo de sustancias $y$ conductas sexuales de riesgo

Se analizó la relación entre distrés psicológico y CS, CSR y CS-CSR. Al efecto, se dividió a los sujetos por el grado de malestar psicológico que reportaron en la escala considerando dos categorías: con bajo distrés, aquellos que puntuaron por debajo del percentil 90; con alto distrés, aquellos que puntuaron a partir del percentil 90 y más. Con respecto al CS los sujetos fueron divididos por consumo o no consumo de tabaco, alcohol y drogas (incluyendo marihuana, cocaína y éxtasis). Los resultados obtenidos mediante el procedimiento de $\chi^{2}$ se muestran en la Tabla 6. Con respecto a CSR y CS-CSR se clasificó a los sujetos según presentaban o no CSR (actividad sexual con parejas ocasionales y no uso del condón) o CS-CSR (tener sexo bajo el influjo del alcohol y tener sexo bajo el influjo de drogas), con los resultados descritos en la Tabla 7. Ambas tablas muestran las proporciones concernientes a sujetos con alto distrés y presencia o no de la conducta en estudio (CS, CSR o CS-CSR según el caso).
Con respecto al CS se detectaron más diferencias entre las mujeres que entre los hombres prácticamente en todas las subescalas; al parecer, la proporción de mujeres bajo fuerte distrés que recurren al CS es mayor que la proporción que no lo hace, especialmente en lo que respecta al consumo de alcohol y drogas. La proporción de mujeres con malestar psicológico intenso es mayor en el caso del consumo de drogas que en cualquiera de las otras dos categorías de consumo. Por su parte, los hombres no reportan diferencias con respecto al consumo de alcohol y muy pocas en relación con el tabaco y las drogas, aunque para este último grupo de sustancias se puede apreciar un comportamiento similar al de las mujeres: la proporción de hombres con alto distrés que recurren a las drogas es más alto.

Por otro lado, con respecto a CSR los resultados mostraron que la proporción de mujeres con alto distrés psicológico que mantienen relaciones sexuales bajo el influjo de drogas o alcohol es mayor que aquellas que no practican tales conductas. La ansiedad, la depresión, la hostilidad y la ideación 
TABLA 6

Comparación entre sujetos con alto y bajo distrés y consumo y no consumo de drogas, tabaco y alcohol

\begin{tabular}{|c|c|c|c|c|c|c|c|c|c|}
\hline \multirow{2}{*}{$\begin{array}{l}\text { Escalas } \\
\text { SCL-90-R }\end{array}$} & \multicolumn{3}{|c|}{$\begin{array}{c}\text { Drogas } \\
\text { ¿Consume? }\end{array}$} & \multicolumn{3}{|c|}{$\begin{array}{c}\text { Tabaco } \\
\text { ¿Consume? }\end{array}$} & \multicolumn{3}{|c|}{$\begin{array}{c}\text { Alcohol } \\
\text { ¿Consume? }\end{array}$} \\
\hline & SÍ & $\mathrm{NO}$ & $\chi^{2}$ & SÍ & $\mathrm{NO}$ & $x^{2}$ & SÍ & $\mathrm{NO}$ & $\chi^{2}$ \\
\hline \multicolumn{10}{|l|}{ MUJERES } \\
\hline Obses. comp. & 38 & 20.8 & $8.35 * *$ & & & & 27.5 & 14.3 & $3.74^{*}$ \\
\hline Sensi. inter. & 33.8 & 21.3 & $4.50 *$ & 29.3 & 18.2 & $4.57 *$ & 27.1 & 12.2 & $4.80 *$ \\
\hline Depresión & 35.2 & 20.3 & $6.43 * *$ & & & & 27.1 & 10.2 & $6.28 * *$ \\
\hline Ansiedad & 25.8 & 9.4 & $8.51^{* * *}$ & & & & 24.5 & 10.2 & $4.78^{*}$ \\
\hline Hostilidad & 31 & 15.5 & $8.14 * *$ & 23.6 & 14 & $3.95 *$ & 21.8 & 8.2 & $4.82 *$ \\
\hline Ans. Fóbica & 35.2 & 20.3 & $6.43 * *$ & & & & & & \\
\hline Idea.paranoi. & 32.4 & 17.4 & $7.11^{* * *}$ & & & & 24.5 & 6.1 & $8.11^{* * *}$ \\
\hline Psicoticismo & 31.9 & 17.4 & $5.91 * *$ & 24.8 & 15.7 & $3.45^{*}$ & 23.6 & 8.2 & $5.81 * *$ \\
\hline Distres (ISG) & 35.2 & 18.8 & $7.99 * *$ & 28.7 & 15.7 & $6.47 * *$ & 25.8 & 10.2 & $5.51 * *$ \\
\hline \multicolumn{10}{|l|}{ HOMBRES } \\
\hline Obses. comp. & 24.2 & 8.5 & $8.25 * *$ & 17.9 & 7.5 & $3.77^{*}$ & & & \\
\hline Ansiedad & 25.8 & 9.4 & $8.51^{* *}$ & 28 & 14 & $7.81^{*}$ & & & \\
\hline Hostilidad & 29 & 14.5 & $5.34 *$ & 24.1 & 19.6 & $3.92 *$ & & & \\
\hline Ans. Fóbica & 29 & 14.5 & $5.41 *$ & & & & & & \\
\hline
\end{tabular}

$* p<0.05 ; * * p<0.01 ; \chi 2=$ chi cuadrado

Nota. Solo se incluyen relaciones significativas.

Fuente: elaboración propia.

paranoide parecen jugar un papel importante en estos casos.

A diferencia de las mujeres, la proporción de los hombres con alto malestar psicológico que establecen relaciones sexuales con parejas ocasionales es prácticamente el doble de aquellos que no lo hacen; el miedo y la soledad derivados de las dimensiones de ansiedad fóbica, ideación paranoide y psicoticismo, podrían jugar un papel impulsor de esta conducta. Para el resto de las CSR no se encontraron diferencias en este género.

Llamó la atención que las proporciones de hombres y mujeres que utilizan o no el preservativo no mostraron diferencias significativas, lo cual podría sugerir que el distrés juega un papel poco definitorio en esta CSR.

En conjunto, estos resultados parecen indicar la utilidad del SCL-90-R para discriminar entre sujetos con alto nivel de distrés que recurren al CS o a las CSR de aquellos que bajo esta misma condición de distrés no lo hacen.

\section{Discusión}

Los resultados reportados para la presente investigación han mostrado coincidencias con respecto a lo hallado tanto en muestras mexicanas como en muestras de otras latitudes. La estructura factorial propuesta por Derogatis (1994) y Derogatis y Cleary (1977a, 1977b) no pudo ser replicada. La muestra de estudiantes universitarios utilizada arrojó 18 factores que explicaron el $63.1 \%$ de la varianza; resultados similares a los obtenidos por Caparrós et al. (2007) en España, quienes arribaron a una estructura de 19 factores que explicaron el $58 \%$ de la varianza. Otros investigadores han reportado estructuras factoriales diferentes para el SCL-90-R, habiéndose sugerido (Vassend \& Skrondal, 1999 citado por Gempp \& Avendaño, 2008) que tales inconsistencias podrían deberse al tipo de ítems utilizados en el instrumento. Parece ser, según lo reportado, que estos mantienen distribuciones y correlaciones que dependen 
TABLA 7

Comparación entre sujetos con alto y bajo distrés psicológico y conductas sexuales de riesgo por sexo

\begin{tabular}{|c|c|c|c|c|c|c|c|c|c|c|c|c|}
\hline \multirow{2}{*}{$\begin{array}{c}\begin{array}{c}\text { Escalas } \\
\text { SCL-90-R }\end{array} \\
\text { MUJERES }\end{array}$} & \multicolumn{3}{|c|}{$\begin{array}{l}\text { Actividad sexual con } \\
\text { parejas ocasionales }\end{array}$} & \multicolumn{3}{|c|}{ Uso del condón } & \multicolumn{3}{|c|}{ Sexo y Alcohol } & \multicolumn{3}{|c|}{ Sexo y Drogas } \\
\hline & $\mathrm{C}$ & $\mathrm{S}$ & $\chi^{2}$ & $\mathrm{C}$ & $\mathrm{S}$ & $c^{2}$ & $\mathrm{C}$ & $S$ & $\chi^{2}$ & $\mathrm{C}$ & $\mathrm{S}$ & $\chi^{2}$ \\
\hline Somatización & & & & & & & & & & 45.8 & 18.2 & $8.2^{* *}$ \\
\hline Obses. Comp. & & & & & & & 32.8 & 16.2 & $8.1^{* *}$ & 54.2 & 21.7 & $12 * *$ \\
\hline Depresión & & & & & & & 29.7 & 16.2 & $5.6^{*}$ & 41.7 & 21.7 & $4.7^{*}$ \\
\hline Ansiedad & & & & & & & 28.1 & 17.2 & $3.7 *$ & 50 & 29.2 & $10.6 * *$ \\
\hline Hostilidad & & & & & & & 28.1 & 11.1 & $9.8^{* *}$ & & & \\
\hline Ans. Fóbica & & & & & & & & & & 50 & 21.7 & $9.3^{* *}$ \\
\hline Idea. Paranoi. & & & & & & & 25 & 15.2 & $3.3^{*}$ & 54.2 & 16.7 & $18.3^{* *}$ \\
\hline Psicoticismo & 31.8 & 18 & $4.5^{*}$ & & & & & & & & & \\
\hline Distrés (ISG) & & & & 26.2 & 13.5 & $3.6^{*}$ & 30.5 & 14.1 & $8.3^{* *}$ & 54.2 & 19.7 & $14.2^{* * *}$ \\
\hline \multicolumn{13}{|l|}{ HOMBRES } \\
\hline Ans. Fóbica & 24.4 & 12 & $4 *$ & & & & & & & & & \\
\hline Idea. Paranoi. & 23.2 & 9.8 & $5.3^{*}$ & & & & & & & & & \\
\hline Psicoticismo & 24.4 & 9.8 & $6.2^{*}$ & & & & & & & & & \\
\hline Distrés (ISG) & 19.5 & 8.5 & $4 *$ & & & & & & & & & \\
\hline
\end{tabular}

Nota. C y $S=$ Proporción (\%), Con y Sin conducta sexual de riesgo respectivamente. ${ }^{*} p<0.05 ; * * p<0.01$.

Fuente: elaboración propia.

de la prevalencia específica en cada población evaluada.

Las altas correlaciones obtenidas entre las dimensiones primarias del SCL-90-R (de 0.5 y de hasta 0.81) aportan evidencia con respecto a un importante sentido ya expuesto por otros investigadores: el SCL-90-R podría ser considerado como una medida unidimensional de psicopatología general (Martínez-Azumendi, Fernandez-Gómez \& Beitia-Fernández, 2001) o de distrés general (Caparrós et al., 2007).

Por otro lado, la consistencia interna de la escala, estructurada conforme a los criterios de Derogatis y colaboradores en la muestra explorada, fue muy similar en prácticamente todas las dimensiones y el índice de severidad global a las reportadas por varios investigadores para muestras mexicanas, de España, Argentina y Chile (cf., Bonicatto, Dew, Soria \& Seghezzo, 1997; Caparrós et al., 2007; Cruz et al., 2005; Gempp \& Avendaño, 2008; Lara et al., 2005). Parece ser que el SCL-90-R posee una consistencia interna óptima y reproducible en distintas latitudes, lo cual puede hacer de este instrumento, al menos en términos de confiabilidad, una opción adecuada para ser usado en estudios que contemplen análisis interculturales.

En general, se pudo observar una cierta tendencia en los hombres a puntuar más bajo que las mujeres en todas las dimensiones, aunque no siempre de manera significativa. Las diferencias por sexo halladas en la investigación parecen confirmar los resultados reportados por Cruz et al. (2005) en una muestra de sujetos mexicanos que incluyó estudiantes universitarios además de personal médico y familiares de pacientes. Ambos trabajos coincidieron en hallar diferencias significativas en las subescalas de Somatización y Depresión, pero difirieron en Sensibilidad interpersonal donde Cruz et al. encontraron diferencias, y Obsesivo compulsivo y el Índice de severidad global donde la presente investigación las halló; el resto de las dimensiones no reportaron diferencias significativas en ambos trabajos. Otros investigadores en Argentina y España obtuvieron diferencias significativas por sexo 
con las mujeres, alcanzando puntuaciones más altas que los hombres en prácticamente todas las dimensiones excepto Hostilidad (muestras argentinas) e Ideación paranoide (muestras argentinas y española; Bonicatto et al., 1997; Caparrós et al., 2007; Casullo, 2004a). La constante en todas estas investigaciones parece ser cierta tendencia en las mujeres a puntuar más alto que los hombres en esta medida de distrés.

Como han sugerido Gempp y Avendaño (2008), es importante realizar estudios que incluyan como objetivo de estudio el comportamiento de la escala en distintas culturas hispanoparlantes. Otro punto para considerar, es el empleo de un formato de escala de aplicación universal, es decir, aplicable en diferentes culturas iberoamericanas sin variaciones. Generalmente, las investigaciones que han adaptado el SCL-90-R a poblaciones específicas no incluyen su versión dentro del artículo que difunde sus resultados, lo cual no añade claridad al asunto. La escala utilizada en este trabajo ha sido validada en México por Cruz et al. (2005), coincidiendo ampliamente con la propuesta por Casullo (2004a) en sujetos argentinos. Esencialmente, la única diferencia es el empleo de términos adaptados al uso popular. En todo caso, se sugiere el uso de la escala utilizada en esta investigación. El SCL-90-R parece poseer cualidades adecuadas para ser empleado en sujetos mexicanos. Los resultados reportados en la presente investigación pueden ser generalizables a sujetos con una edad entre los 18 y los 26 años de edad, preferentemente universitarios y mexicanos. Bonicatto et al. (1997) han sugerido la conveniencia de desarrollar y usar datos normativos basados en muestras de las localidades en que se desea evaluar. Al efecto, para la interpretación de la escala, toda vez que sea aplicada a una muestra similar a la empleada aquí, se sugiere la utilización de los datos normativos descritos en este trabajo, lo cual no elimina la conveniencia de desarrollar criterios para otros subgrupos mexicanos; como ha sido posible mostrar aquí, variaciones en la composición de la muestra utilizada pueden generar resultados sensiblemente diferentes (cf., Cruz et al., 2005).

\section{Referencias}

Antón, R. F. A. \& Espada, J. P. (2009). Consumo de sustancias y conductas sexuales de riesgo para la transmisión del VIH en una muestra de estudiantes universitarios. Anales de Psicología, 25(2), 344-350.

Bonicatto, S., Dew, M. A., Soria, J. J. \& Seghezzo, M. E. (1997). Validaty and reliability of Symptom Checklist 90 (SCL90) in an Argentine population sample. Social Psychiatry and Psychiatric Epidemio$\log$, 32(6), 332-338.

Bonynge, E. R. (1993). Unidimensionality of SCL-90-R scales in adult and adolescent crisis samples. Journal of Clinical Psychology, 49(2), 212-215.

Brodbeck, J., Vilén, U. L., Bachmann, M., Znoj, H. \& Alsaker, F. (2010). Sexual risk behavior in emerging adults: Gender-specific effects of hedonism, psychosocial distress, and sociocognitive variables in a 5-year longitudinal study. AIDS Educational and Prevention, 22(2), 148-159.

Caparrós, C. B., Villar, H. E. Ferrer, J. J. \& Viñas, P. F. (2007). Sympton Check-List 90-R: fiabilidad, datos normativos y estructura factorial en estudiantes universitarios. International Journal of Clinical and Health Psychology, 7(3), 781-794.

Caraveo-Anduaga, J. J. \& Colmenares, B. E. (2002). Los trastornos psiquiátricos y el abuso de sustancias en México: panorama epidemiológico. Salud Mental, 25(2), 9-15.

Carrasco, O. M. A., Sánchez, M. V., Ciccotelli, H. \& Del Barrio, B. (2003). Listado de síntomas SCL-90-R: análisis de su comportamiento en una muestra clínica. Acción Psicológica, 2(2), 149-161.

Casullo, M. (2004a). El inventario de síntomas SCL90-R de L. Derogatis. Recuperado el 3 de agosto de 2010, de http://bscw.rediris.es/pub/bscw. cgi/d531850/EL\%20INVENTARIO\%20DE\%20 S\%C3\%8DNTOMAS\%20SCL-90-R\%20de\%20 L.\%20Derogatis.pdf

Casullo, M. (2004b). Síntomas psicopatológicos en adultos urbanos. Psicología y Ciencia Social, 6(1), 49-57.

Cruz, F. C. S., López, B. L., Blas, G. C., González, M. L. \& Chávez, B. R. A. (2005). Datos sobre la validez y confiabilidad de la Symptom Check List 90 (SCL 
90) en una muestra de sujetos mexicanos. Salud Mental, 28(1), 72-81.

Derogatis, L. (1994). SCL 90-R administration, scoring and procedures manual (3a. ed.). Minneapolis: $\mathrm{Na}$ tional Computer Systems.

Derogatis, L. \& Cleary, P. (1977a). Confirmation of dimensional structure of the SCL-90: A study in construct validation. Journal of Clinical Psychology, 33(4), 981-982.

Derogatis, L. \& Cleary, P. (1977b). Factorial invariance across gender for the primary symptoms dimension of the SCL-90. British Journal for Social \& Clinical Psychology, 16(4), 347-356.

Derogatis, L., Lipman, R. \& Covi, L. (1973). SCL-90: An outpatient psychiatric rating scale-preliminary report. Psychopharmacology Bulletin, 9(1), 13-28.

Gempp, F. R. \& Avendaño, B. C. (2008). Datos normativos y propiedades psicométricas del SCL-90-R en estudiantes universitarios chilenos. Terapia Psicológica, 26(1), 39-58.

Halkitis, P. N., Mukherjee, P. P. \& Palamar, J. J. (2007). Multi-level modeling to explain methamphetamine use among gay and bisexual men. Addiction, 102(Suppl. 1), 76-83.

Kaplan, M. S. \& Krueger, R. B. (2010). Diagnosis, assessment and treatment of hypersexuality. Journal of Sex Research, 47(2-3), 181-198.

Lara, M. C., Espinosa, S. I., Cárdenas, M. L., Fócil, M. \& Cavazos, J. (2005). Confiabilidad y validez de la SCL-90 en la evaluación de psicopatología en mujeres. Salud Mental, 28(3), 42-50.

Martínez-Azumendi, O., Fernandez-Gómez, C. \& Beitia-Fernández, M. (2001). Variabilidad factorial del SCL-90-R en una muestra psiquiátrica ambulatoria. Actas Españolas de Psiquiatría, 29(2), 95-102.

Meave, S. F. (2008). Relación entre rasgos de personalidad y conductas sexuales de riesgo, elementos para la prevención con adolescentes escolares. Disertación doctoral, Universidad Nacional Autónoma de México, Ciudad de México, México.

Medina-Mora, M. E., Borges, G., Lara, M. C., Benjet, C., Blanco, J. J., Fleiz, B. C., et al. (2003). Prevalencia de trastornos mentales y uso de servicios: resultados de la Encuesta Nacional de Epidemiología Psiquiátrica en México. Salud Mental, 26(4), 1-16. Ortíz, A., Soriano, A., Meza, D., Martínez, R. \& Galván, J. (2006). Uso de sustancias entre hombres y mujeres, semejanzas y diferencias. Resultados del sistema de reporte de información en drogas. Salud Mental, 29(5), 32-37.

Rodríguez, J. \& Hernández, E. (2005). Análisis de la confiabilidad y validez de constructo de un instrumento que mide el consumo de drogas lícitas e ilícitas en estudiantes universitarios. Terapia Psicológica, 23(1), 83-90.

Schulenberg, J. E., Bryant, A. L. \& O'Malley, P. M. (2004). Taking hold of some kind of life: How developmental tasks relate to trajectories of well-being during the transition to adulthood. Developmental \& Psychopatology, 16(4), 1119-1140.

Schwarzwald, J., Weisenberg, M. \& Solomon, Z. (1991). Factor invariance of SCL-90-R: The case of combat stress reaction. Psychological Assessment: A Journal of Consulting and Clinical Psychology, 3(3), 385-390.

Semple, S. J., Strathdee, S. A., Zians, J. \& Patterson, T. L. (2010). Life events and sexual risk among HIVnegative, heterosexual, methamphetamine users. Journal of Sex Research, 47(4), 355-363.

Steer, R. A., Clark, D. A. \& Ranieri, W. F. (1994). Symptom dimensions of the SCL-90-R: A test of the tripartite model of anxiety and depression. Journal of Personality Assessment, 62(3), 525-536.

Uribe, I. (2007). Factores individuales, sociales y culturales asociados a la conducta sexual adolescente. Disertación doctoral, Universidad Nacional Autónoma de México, Ciudad de México, México.

Villatoro,V. J. A., Gutiérrez, L. M. L., Quiroz, V. N., Moreno, L. M., Gaytán, L. L., Gaytán, F. F. I., et al. (2006). Encuesta de estudiantes de la Ciudad de México 2006. Prevalencias y evolución del consumo de drogas. Salud Mental, 32(4), 287-297. 
